\title{
Optical Pattern Fabrication in Amorphous Silicon Carbide with High-Energy Focused Ion Beams
}

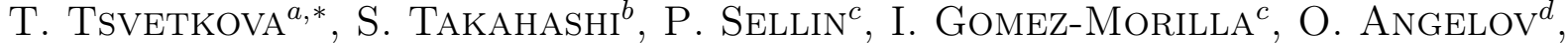 \\ D. Dimova-Malinovska ${ }^{d}$ And J. ZuK ${ }^{e}$ \\ ${ }^{a}$ Institute of Solid State Physics, Bulgarian Academy of Sciences \\ 72 Tzarigradsko Chaussee Blvd., 1784 Sofia, Bulgaria \\ ${ }^{b}$ Centre for Nanostructured Media, School of Mathematics and Physics, The Queen's University of Belfast \\ Belfast BT7 1NN, UK \\ ${ }^{c}$ Surrey Ion Beam Centre, Advanced Technology Institute, School of Electronics and Physical Sciences \\ University of Surrey, Guildford, Surrey GU2 7XH, UK \\ ${ }^{d}$ Central Laboratory for Solar Energy and New Energy Sources, Bulgarian Academy of Sciences \\ 72 Tzarigradsko Chaussee Blvd., 1784 Sofia, Bulgaria \\ ${ }^{e}$ Institute of Physics, Maria Curie-Skłodowska University, pl. M. Curie-Skłodowskiej 1, 20-031 Lublin, Poland \\ Topographic and optical patterns have been fabricated in a-SiC films with a focused high-energy $(1 \mathrm{MeV}) \mathrm{H}^{+}$ \\ and $\mathrm{He}^{+}$ion beam and examined with near-field techniques. The patterns have been characterized with atomic \\ force microscopy and scanning near-field optical microscopy to reveal local topography and optical absorption \\ changes as a result of the focused high-energy ion beam induced modification. Apart of a considerable thickness \\ change (thinning tendency), which has been observed in the ion-irradiated areas, the near-field measurements \\ confirm increases of optical absorption in these areas. Although the size of the fabricated optical patterns is in \\ the micron-scale, the present development of the technique allows in principle writing optical patterns up to the \\ nanoscale (several tens of nanometers). The observed values of the optical contrast modulation are sufficient to \\ justify the efficiency of the method for optical data recording using high-energy focused ion beams.
}

PACS: 41.75.Ak, 42.70.Ln, 68.37.Uv, 73.61.Jc

\section{Introduction}

This paper is related to a new approach of recording archival information using high-energy focused $\mathrm{He}^{+}$ ion beams for optical patterning of thin amorphous hydrogenated silicon carbide (a-SiC:H) alloy films. These materials have been studied for many years for their wide range of useful optical properties (e.g. high transparency in the visible region due to their wide optical band gap), as well as mechanical durability and chemical inertness [1]. These properties, together with thermal stability and ease of fabrication, make a-SiC:H a very promising candidate for various optoelectronic device applications in adverse environments $[2,3]$. Radio-frequency $(\mathrm{RF})$ reactive magnetron sputtering is a convenient method for preparation of a-SiC:H films where, as is often the case, low density-of-states material is not required [4].

\footnotetext{
* corresponding author; e-mail: tania_tsvetkova@yahoo.co.uk
}

The use of ion beam techniques is a very attractive method to control material properties $[5,6]$. Recently, using the emerging technology of ion micro-beams [7], promising results have been obtained for the possible application of thin a-SiC:H films in high-density archival data storage $[8,9]$. This approach has been further developed by implementing ion doping with focused ion beam (FIB) systems that use $\mathrm{Ga}^{+}$or other chemically active ion species [10-13]. Still another approach of obtaining optical contrast in a-SiC:H films, suitable for such applications, is the use of high-energy $(\mathrm{MeV})$ focused ion beams via structural modification of the irradiated film material $[14,15]$.

In order to characterize the optical properties of sub-micron structures, a "super-resolution" technique is required. In addition, ion irradiation may cause ablation of the a-SiC:H, leading to changes of the topographic features of the surface. Therefore a technique to map the topographic features of the patterns, such as atomic force microscopy (AFM) $[16,17]$ is also necessary to characterize the modified surfaces. From these points of view, 
scanning near-field optical microscopy (SNOM) [17-19], with the shear force technique that provides topographic data, is a promising method that can obtain both optical and topographic images of the sample simultaneously.

In the present work, a focused high-energy $\mathrm{He}^{+}$ion beam has been used to create micron-scale optical patterns in a-SiC:H films. The patterning process has been studied by applying AFM and SNOM to investigate topographic as well as optical variation of the modified surface.

\section{Experimental}

The a-Si ${ }_{1-x} \mathrm{C}_{x}: \mathrm{H}$ films were deposited onto glass substrates by $\mathrm{RF}(13.56 \mathrm{MHz})$ reactive magnetron sputtering. A composite target, comprising a monocrystalline (100) silicon wafer with chips of pure graphite, placed on it, with two different graphite to silicon area ratios (0.025 and 0.25) was sputtered in an argon-hydrogen gas mixture. The deposited in this way amorphous films differ in carbon content, $x$, which was estimated by the Rutherford backscattering (RBS) analysis to be 0.18 and 0.35 , respectively. The film thickness was determined by Talystep profilometry and optical data analysis to be $\approx 1 \mu \mathrm{m}$.

Focused $\mathrm{He}^{+}$-irradiation in these samples was performed with ion beam energy $E=1 \mathrm{MeV}$ and ion dose $D=1.6 \times 10^{16} \mathrm{~cm}^{-2}$ for preparing patterned a-Si $\mathrm{Si}_{1-x} \mathrm{C}_{x}: \mathrm{H}$ samples (Fig. 1). Optical patterns in the form of series of lines were produced with two different line-widths $20 \mu \mathrm{m}$ (Fig. 1a) and $2 \mu \mathrm{m}$ (Fig. 1b). The patterns were studied in detail by near-field techniques, like AFM and SNOM. Atomic force microscope (Dimension 3000 Digital Instruments) was used to analyze the topography of the fabricated patterned samples. The custom-built scanning near-field optical microscope was used to study the changes in the local absorption of the irradiated (patterned) areas as compared to the non-irradiated ones.

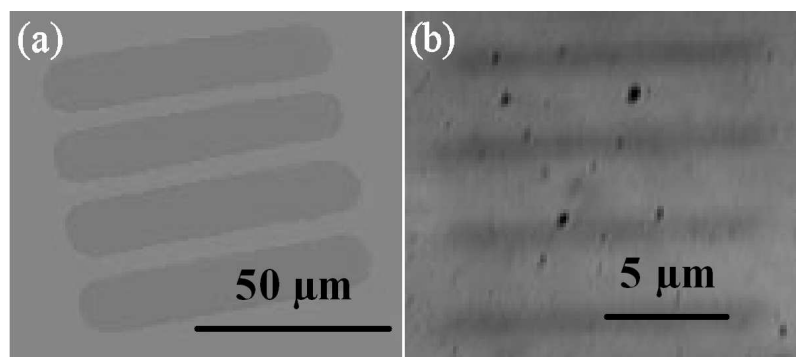

Fig. 1. Microphotograph of optical patterns written with high energy focused $\mathrm{He}^{+}$beam $(E=1 \mathrm{MeV}$, $\left.D=1.6 \times 10^{16} \mathrm{~cm}^{-2}\right)$ in a-SiC:H films in the form of 4 lines with linewidth: $20 \mu \mathrm{m}$ (a) and $2 \mu \mathrm{m}$ (b).

The details of the SNOM measurements are described in our previous work $[11,12]$. Briefly, an unpolarized beam from a He-Ne laser (633 nm) illuminates the substrate side of the sample. The laser is not focused in order to keep the laser intensity on the scanned area as uniform as possible. Light passing through the patterned area is collected by a sharp, uncoated optical fibre probe from one point to the next, in order to form optical near-field images. The gap between the probe end and the sample is kept constant with a non-optical shear-force technique that employs a quartz tuning fork; feedback from the shear-force regulation scheme yields topographic information. Topography and local optical mapping are thus achieved simultaneously.

\section{Results and discussion}

The obtained optical patterns in a-SiC:H by high-energy focused $\mathrm{He}^{+}$ion beams are the first known result of registering such patterns based mainly on the electronic stopping mechanism in the implanted films $[14,15]$, unlike the two previous known cases where heavy ions induced radiation defects [7-9] or chemical modification mechanisms [10-13] were shown to define the process. Therefore, studying the patterns in detail by near-field techniques, like AFM and SNOM, are expected to supply details of the ion beam induced modification processes and further elucidate the mechanism of the observed optical contrast obtained by high energy focused $\mathrm{He}^{+}$implantation in a-SiC:H.

Patterned a-SiC:H samples, implanted with high energy focused $\mathrm{He}^{+}$beam $(E=1 \mathrm{MeV}, D=1.6 \times$ $10^{16} \mathrm{~cm}^{-2}$ ), were studied by AFM. The AFM results for the optical pattern shown in Fig. 1a are presented in Fig. 2. The topographic features of a line $20 \mu \mathrm{m}$ wide and the same line rotated at $45^{\circ}$ are shown in Fig. 2a and $b$, respectively. The AFM analysis reveals that the irradiated areas are topographically lower than the non-irradiated ones. This is supposedly due to the ablation of the film caused by the ion beam. In Fig. 3, a cross-sectional view of the AFM image of the line shown in Fig. 2 is presented. The observed decrease in the film thickness $(10-15 \mathrm{~nm})$ is assumed to result from a mechanism of high local temperature increase and ion beam assisted vaporization/ablation of the top surface of the film.

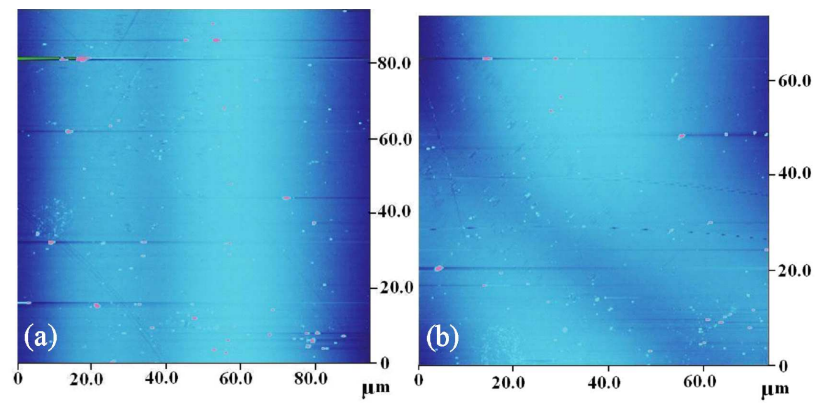

Fig. 2. Topographic AFM image of a single line pattern with linewidth $20 \mu \mathrm{m}$ written with high energy focused $\mathrm{He}^{+}$beam $\left(E=1 \mathrm{MeV}, D=1.6 \times 10^{16} \mathrm{~cm}^{-2}\right)$ in a-SiC:H film (a) and the same rotated $45^{\circ}$ (b). 


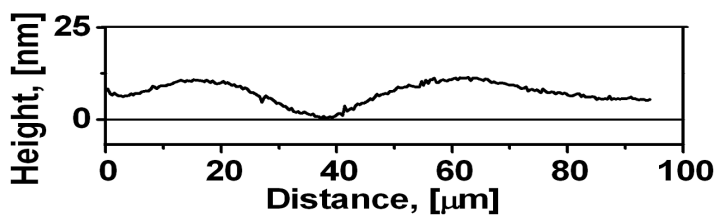

Fig. 3. AFM cross-sectional view of a single line pattern with linewidth $20 \mu \mathrm{m}$ written with high energy focused $\mathrm{He}^{+}$beam $\left(E=1 \mathrm{MeV}, D=1.6 \times 10^{16} \mathrm{~cm}^{-2}\right)$ in a-SiC:H film.

As mentioned in the introduction, SNOM can provide an optical image of the corresponding topographic image simultaneously. The optical pattern shown in Fig. 1b, produced by high energy focused $\mathrm{He}^{+}$beam $(E=1 \mathrm{MeV}$, $D=1.6 \times 10^{16} \mathrm{~cm}^{-2}$ ) in an a-SiC:H film in the form of 4 lines with $2 \mu \mathrm{m}$ width each, is investigated with SNOM as a demonstration of its potentiality. The obtained topographic and corresponding optical images are demonstrated in Fig. 4. Compared with un-patterned areas, e.g., in the top side of the image, the areas irradiated with ion beam are topographically lower, whereas they are optically more opaque. The trend of the topographic features of the irradiated areas is the same as that obtained with AFM, and the trend of optical contrast obtained with SNOM is qualitatively the same as that obtained with conventional optical microscopy, shown in Fig. 1b.
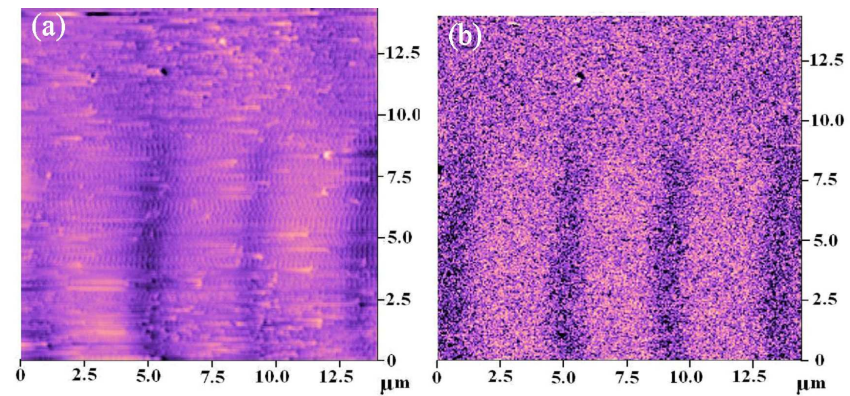

Fig. 4. SNOM produced topographic image (a) and corresponding near-field optical contrast image (b) of an optical pattern written with high energy focused $\mathrm{He}^{+}$ beam $\left(E=1 \mathrm{MeV}, D=1.6 \times 10^{16} \mathrm{~cm}^{-2}\right)$ in a-SiC:H film in the form of 4 lines with linewidth $2 \mu \mathrm{m}$.

Typical cross-sectional views of the SNOM topographic and optical images shown in Fig. 4 are presented in Fig. 5. In Fig. 5a the cross-sectional view of the topographic image is shown, representing again that the irradiated areas are topographically lower. Figure 5b shows the cross-sectional view of the optical image, demonstrating the increased optical absorption in the irradiated areas as compared to the non-irradiated, even though the thickness of the film in these areas is decreased at the same time.

The AFM and SNOM data both confirm that the implanted areas thickness decreases. The results thus differ from previous data on low-energy focused ion bom-

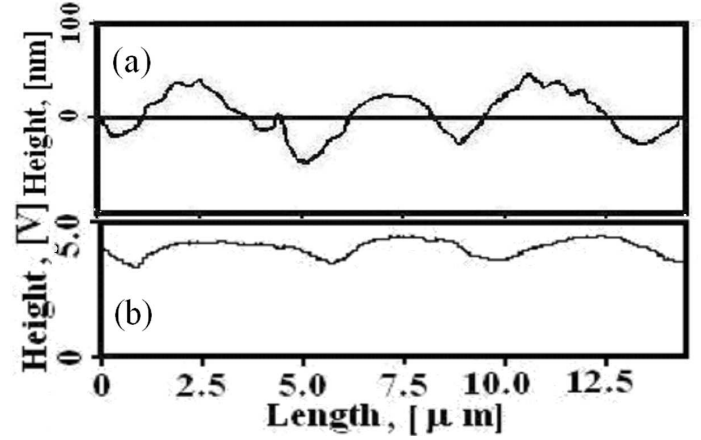

Fig. 5. Cross-sectional analysis of the SNOM image in a topographic mode (a) and corresponding near-field optical contrast mode (b) of the optical pattern written with high energy focused $\mathrm{He}^{+}$beam $(E=1 \mathrm{MeV}$, $\left.D=1.6 \times 10^{16} \mathrm{~cm}^{-2}\right)$ in a-SiC:H film in the form of 4 lines with linewidth $2 \mu \mathrm{m}$.

bardment effects in diamond-like carbon films, where bombardment induced graphitization results in surface expansion, i.e. thickness increase in the irradiated areas [20]. The observed different topography change in our case is assumed to be the result of the higher energy deposition in the implanted pattern area and accompanying ion beam assisted ablation mechanism.

\section{Conclusion}

Micron-scale patterning of a-SiC:H films using high energy focused $\mathrm{He}^{+}$beam $(E=1 \mathrm{MeV}, D=1.6 \times$ $10^{16} \mathrm{~cm}^{-2}$ ) has been successfully demonstrated. SNOM has been used as a versatile tool to characterize the topographic and optical profiles of ion implanted a-SiC:H. By mapping topographic and optical contrast simultaneously, it has been proved that the irradiation with $\mathrm{He}^{+}$ ions causes surface thinning, in addition to the increase of optical density associated with the irradiation induced structural modification of the film. This result is consistent with data obtained using conventional optical microscopy and AFM. Although the size of the fabricated optical patterns is in the micron-scale, the present development of the technique allows in principle writing optical patterns up to the nanoscale (several tens of nanometers). Thus the present results are of interest for applications in high-density optical data storage for creating permanent optical archives.

\section{Acknowledgments}

This work was supported in part by EPSRC. T.T. gratefully acknowledges the financial support from the International Centre for Experimental Physics (IRCEP) at Queen's University Belfast, under the Distinguished Visiting Fellowship scheme. 


\section{References}

[1] J. Bullot, M.P. Schmidt, Phys. Status Solidi B 143, 345 (1987).

[2] J.A. Powell, L. Matus, in: Amorphous and Crystalline Silicon Carbide, Eds. G.L. Harris, C.Y.W. Yang, Springer, Berlin 1989.

[3] Amorphous and Microcrystalline Semiconductor Devices, Ed. J. Kanicki, Artech House, Boston 1991.

[4] T. Nagai, H. Yamamoto, I. Kobayashi, J. Phys. E 15, 520 (1982).

[5] J.K. Hirvonen, Ion Implantation and Ion Beam Processing of Materials, North Holland, Amsterdam 1984.

[6] J.F. Ziegler, Ion Implantation, Academic Press, New York 1988.

[7] K. Böhringer, K. Jousten, S. Kalbitzer, Nucl. Instrum. Methods Phys. Res. B 30, 289 (1988).

[8] B. Ruttensperger, G. Krötz, G. Müller, G. Derst, S. Kalbitzer, J. Non-Cryst. Solids 137-138, 635 (1991).

[9] G. Müller, Nucl. Instrum. Methods Phys. Res. B 80-81, 957 (1993).

[10] T. Tsvetkova, in: Beam Processing of Advanced Materials, Eds. J. Singh, S. Copley, J. Mazumder, ASM International, Metals Park 1996, p. 207.

[11] T. Tsvetkova, S. Takahashi, A. Zayats, P. Dawson, R. Turner, L. Bischoff, O. Angelov, D. Dimova-Malinovska, Vacuum 79, 94 (2005).
[12] T. Tsvetkova, S. Takahashi, A. Zayats, P. Dawson, R. Turner, L. Bischoff, O. Angelov, D. Dimova-Malinovska, Vacuum 79, 100 (2005).

[13] S. Takahashi, P. Dawson, A.V. Zayats, L. Bischoff, O. Angelov, D. Dimova-Malinovska, T. Tsvetkova, P.D. Townsend, J. Phys. D, Appl. Phys. 40, 7492 (2007).

[14] T. Tsvetkova, P. Sellin, R. Carius, O. Angelov, D. Dimova-Malinovska, J. Optoelectron. Adv. Mater. 9, 375 (2007)

[15] T. Tsvetkova, P. Sellin, R. Carius, O. Angelov, D. Dimova-Malinovska, J. Zuk, Nucl. Instrum. Methods Phys. Res. B 267, 1583 (2009).

[16] G. Binning, C.F. Quate, Ch. Gerber, Phys. Rev. Lett. 56, 930 (1986).

[17] G. Friedbacher, H. Fuchs, Pure Appl. Chem. 71, 1337 (1999).

[18] Near Field Optics, Eds. D.W. Pohl, D. Courjon, Kluwer Academic Publishers, Dordrecht 1993.

[19] D. Richards, A.V. Zayats, Nano-Optics and Near-Field Microscopy, Eds. D. Richards, A.V. Zayats, Artech House, Boston 2008.

[20] S. Kalbitzer, Nucl. Instrum. Methods Phys. Res. B 218, 343 (2004). 